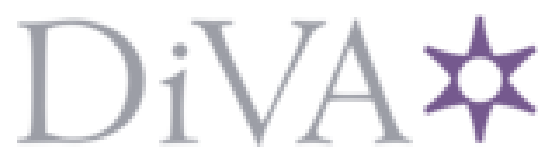

http://www.diva-portal.org

\title{
Postprint
}

This is the accepted version of a paper presented at PowerTech Eindhoven 2015.

Citation for the original published paper:

Adib Murad, A., Gómez, F J., Vanfretti, L. (2015)

Equation-Based Modeling of Three-Winding and Regulating Transformers using Modelica. In: IEEE conference proceedings

http://dx.doi.org/10.1109/PTC.2015.7232503

N.B. When citing this work, cite the original published paper.

Permanent link to this version:

http://urn.kb.se/resolve?urn=urn:nbn:se:kth:diva-171220 


\section{Equation-Based Modeling of Three-Winding and Regulating Transformers using Modelica}

\author{
Mohammed Ahsan Adib Murad, Francisco José Gómez \\ KTH, Royal Institute of Technology, \\ Stockholm, Sweden \\ maamurad@kth.se, fragom@kth.se, luigiv@kth.se
}

\author{
Luigi Vanfretti \\ KTH Royal Institute of Technology, Stockholm, Sweden \\ Statnett SF, Oslo, Norway \\ luigi.vanfretti@kth.se, luigi.vanfretti@statnett.no
}

\begin{abstract}
The simulation of power transformer models is a critical for analyzing the dynamic behavior of power systems, in particular, when considering voltage magnitude or phase regulation controls. This paper reports results of extending the library of transformers in the iTesla Modelica Power Systems Library. Three transformer models have been implemented: a three-winding transformer, an under-load tap changing transformer (ULTC) and a phase shifting transformer (PST). A simple power system test model was also implemented, both in Modelica and PSAT, to assess the performance of the models. Software-to-software validation is carried out against PSAT.
\end{abstract}

Index terms - Modelica, PSAT, Power Transformer, Simulation Software, Power System Simulation.

\section{EXTENDED ABSTRACT}

Adequate modeling of conventional and controllable power transformers allows studying the dynamic behavior of power network under certain different operating conditions. In the literature, classical transformer models are studied [1]. Different transformer models have been developed, each focusing on a particular application or to represent specific physical phenomena. Generally, transformer models are classified according to their application: lightning overvoltage studies or the purpose of elements of the model, e.g., models based on leakage inductance, transmission line modeling, etc.

From the models above, those used in phasor time-domain simulations can be easily implemented using equation-based modeling languages. This kind of languages allows engineers to implement models directly using mathematical equations. The Modelica equation-based modeling language is objectoriented and open-source, which allows model implementation directly from mathematical equations. This is an important characteristic, which implicitly decouples the model from the mathematical solver, thus providing unambiguous simulation results among different tools [2]. The attractive features of this language have been successfully applied in different areas such as the automotive and aerospace industry [3].
European transmission system security is becoming a challenge due to the growing complexities of the panEuropean power network. To overcome these complexities, the FP7 iTESLA (Innovative Tools for Electrical System Security within Large Areas) project was initiated to develop a toolbox that will support the operation of the European transmission network [4]. The iTesla project has adopted the Modelica language for modeling of power system dynamic components, and a Modelica library compatible with Modelica tools has been developed.

The purpose of this work is to improve this Power System Library with the implementation of new Modelica models of conventional power system components (Transformers) for Phasor Time-Domain Simulation. To implement these models the PSAT implementation is taken as reference [5]. Finally, to prove that Modelica models of transformers have the expected behavior, a software-to-software validation is performed, taken PSAT as a software reference for this validation.

\section{IMPLEMENTATION OF TRANSFORMER MODELS}

This work reports the implementation and validation of Modelica models for two regulating transformers and a Three Winding Transformer. The regulating transformers considered herein are: Under Load Tap Changing (ULTC) and Phase Shifting Transformer (PST) transformers. The mathematical description in [5] is taken as reference for model implementation. Due to space limitations, only one model and a summary of the rest of the work are provided in this abstract.

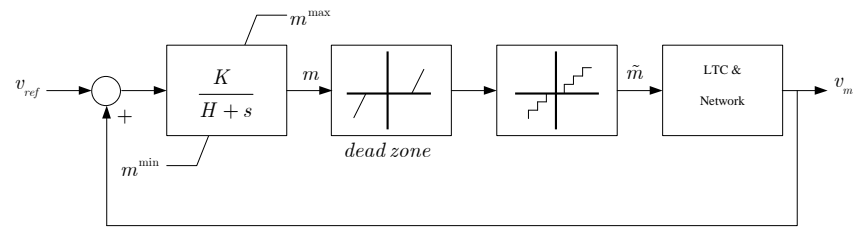

Figure 1: Secondary voltage control scheme of ULTC [14]. 


\section{A. ULTC (Under Load Tap Changer)}

ULTC is a regulating transformer that regulates the voltage or reactive power at the secondary side of the transformer. The regulator used to control the secondary voltage shown in Fig.1. The ULTC transformer is modeled as an equivalent PI circuit as depicted in Fig. 2.

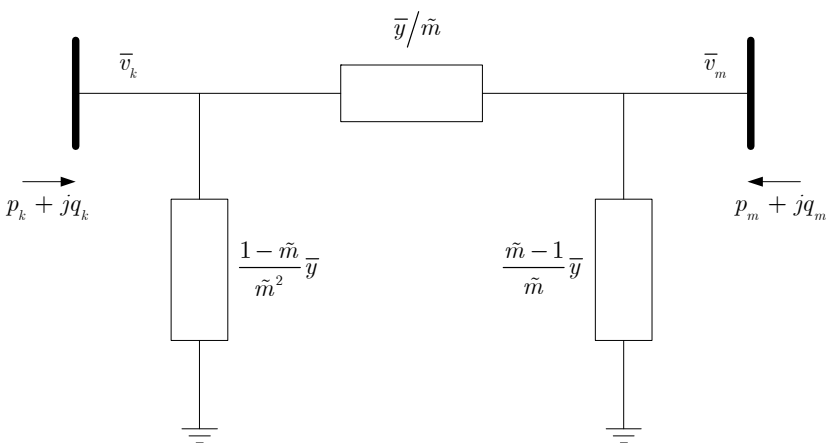

Figure 2: Equivalent pi circuit of ULTC [14].

The current injection at Bus $k\left(i_{k}\right)$ and Bus $m\left(i_{m}\right)$ are calculated from:

$$
\left[\begin{array}{l}
\bar{l}_{k} \\
\bar{l}_{m}
\end{array}\right]=\bar{y}\left[\begin{array}{cc}
\frac{1}{m^{2}} & -\frac{1}{m} \\
-\frac{1}{m} & 1
\end{array}\right]\left[\begin{array}{l}
\bar{v}_{k} \\
\bar{v}_{m}
\end{array}\right]
$$

where, $\bar{y}$ is the series admittance, $m$ is the off nominal tap ratio. The tap ratio $m$ is the output of the regulator shown in Fig. 1. The tap ratio step $\Delta m$ is taken as zero, from where

$$
\widetilde{m}=m
$$

To model the secondary voltage control the differential equation used (calculated from the controller shown in Fig. 1):

$$
\dot{m}=-H m+K\left(v_{m}-v_{r e f}\right)
$$

\section{B. ULTC in Modelica}

The Modelica implementation of the ULTC uses the connector class PwPin [6]. First the parameters are defined, as follows:

parameter Real SystemBase $=100$;

parameter Real Vbus1 $=400000$

"Sending end Bus nominal voltage, change of base"; parameter Real Vbus2 $=100000$

"Receiving end Bus voltage, change of base";

parameter Real $\mathrm{Sn}=100$ "Power rating MVA";

parameter Real $V n=400000$

"Voltage rating, primary side $\mathrm{KV}$ ";

parameter Real fn=50 "Frequency rating $\mathrm{Hz}$ ";

parameter Real $\mathrm{kT}=4$

"Nominal Tap ratio (V1/V2), kV/kV";

parameter Real $\mathrm{H}=0.001$ "Integral deviation, p.u."; parameter Real $\mathrm{K}=0.10$ "Inverse time constant, $1 / \mathrm{s}$ " parameter Real m_max $=0.98$

"Max tap ratio, p.u./p.u.";

parameter Real m_min=0.9785

"Min tap ratio, p.u./p.u.";

parameter Real deltam=@ "Tap ratio step, p.u./p.u."

parameter Real v_ref=1.0 "Reference voltage, p.u."; parameter Real XT $=0.001$

"Transformer Reactance, p.u.";

parameter Real rT=0.1 "Transformer Resistance, p.u. parameter Real $d=0.05$ "Dead zone percentage, \%"; parameter Real vm@=1. 008959700699460

"Init. value of the voltage of the Controlled Bus";

parameter Real $\mathrm{m} \odot=0.98$;

Real m "Tap ratio";

Real vk "Voltage at primary, p.u.".

Real vm "Voltage at secondary p.u.";

Real anglevk "Angle at primary";

Real anglevm "Angle at secondary ";

The parameters used to change the basis are declared as protected variables:

\section{protected}

parameter Real V2= Vn/kT "Secondary voltage";

parameter Real Vb2new=Vbus1*Vbus1;

parameter Real Vb2old $=\mathrm{Vn} * \mathrm{Vn}$;

parameter Real $R=r T^{*}($ Vb2old*SystemBase)/(Vb2new*Sn)

"Transformer Resistance, p.u.";

parameter Real $\mathrm{X}=\mathrm{xT} T^{*}\left(\mathrm{Vb} 2 \mathrm{old}{ }^{*}\right.$ SystemBase $) /\left(\mathrm{Vb} 2 \mathrm{new}{ }^{*} \mathrm{Sn}\right)$

"Transformer Reactance, p.u.";

parameter Real vref=v_ref*(V2/Vbus2);

parameter Real gt $=\mathrm{R} /\left(\mathrm{R}^{\wedge} 2+\mathrm{X}^{\wedge} 2\right)$ "Converting resistan

ce to conductance p.u.";

parameter Real bt $=\left(X /\left(R^{\wedge} 2+X^{\wedge} 2\right)\right)$ "Converting reacta nce to susceptance p.u.";

Then, equations (1) and (3) are added to complete the model: equation

$R^{*} p \cdot i r-X^{*} p \cdot i i=\left(1 / m^{\wedge} 2\right){ }^{*} p \cdot v r-(1 / m) * n \cdot v r$;

$R^{\star} p \cdot i i+X^{\star} p \cdot i r=\left(1 / m^{\wedge} 2\right) * p \cdot v i-(1 / m)^{*} n \cdot v i$;

$R^{*} n \cdot i r-X^{*} n \cdot i i=n \cdot v r-(1 / m) * p \cdot v r$;

$x^{*} n \cdot i r+R^{*} n \cdot i i=n \cdot v i-(1 / m){ }^{*} p \cdot v i$;

$\operatorname{der}(m)=-\left(H^{*} m\right)+K^{*}(v m-v r e f)$;

\section{VALIDATION OF THE ULTC MODEL}

Having the Modelica implementation of the ULTC, a small test power system was implemented both in Modelica and PSAT to perform software-to-software validation.

\section{A. Test System}

Fig. 3 shows the Modelica implementation of a three bus test power system. The same test system was implemented in PSAT. Initial guess values for algebraic and state variables in the Modelica test system were taken from the power flow solution calculated in PSAT.

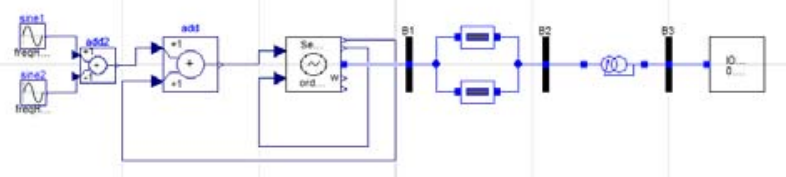

Figure 3: Test system in Modelica to validate ULTC.

\section{B. Validation Case: Perturbations}

To test the dynamic behavior of the ULTC, perturbations are applied introduced in the test system. The perturbations are applied in such a way that the limiters of the ULTC controller are reached. The perturbations applied in this test system are:

Synchronous Machine: Sinusoidal oscillation . $001 * \sin (2 \pi *$ $0.2 * t)$ in the field voltage $\left(v_{f}\right)$ of the generator from time 0 s to $5 \mathrm{~s}$. 
PQ Load: +.05 per unit (pu) reactive power load is added, from $5 \mathrm{~s}$ to $8 \mathrm{~s}$, and $-.05 \mathrm{(pu)}$ reactive power load is added from 8 s to $12 \mathrm{~s}$.

\section{Simulation and Results}

Simulations were performed both with Modelica and PSAT. The outputs of both the software tools match with negligible variation (see Fig. 4).
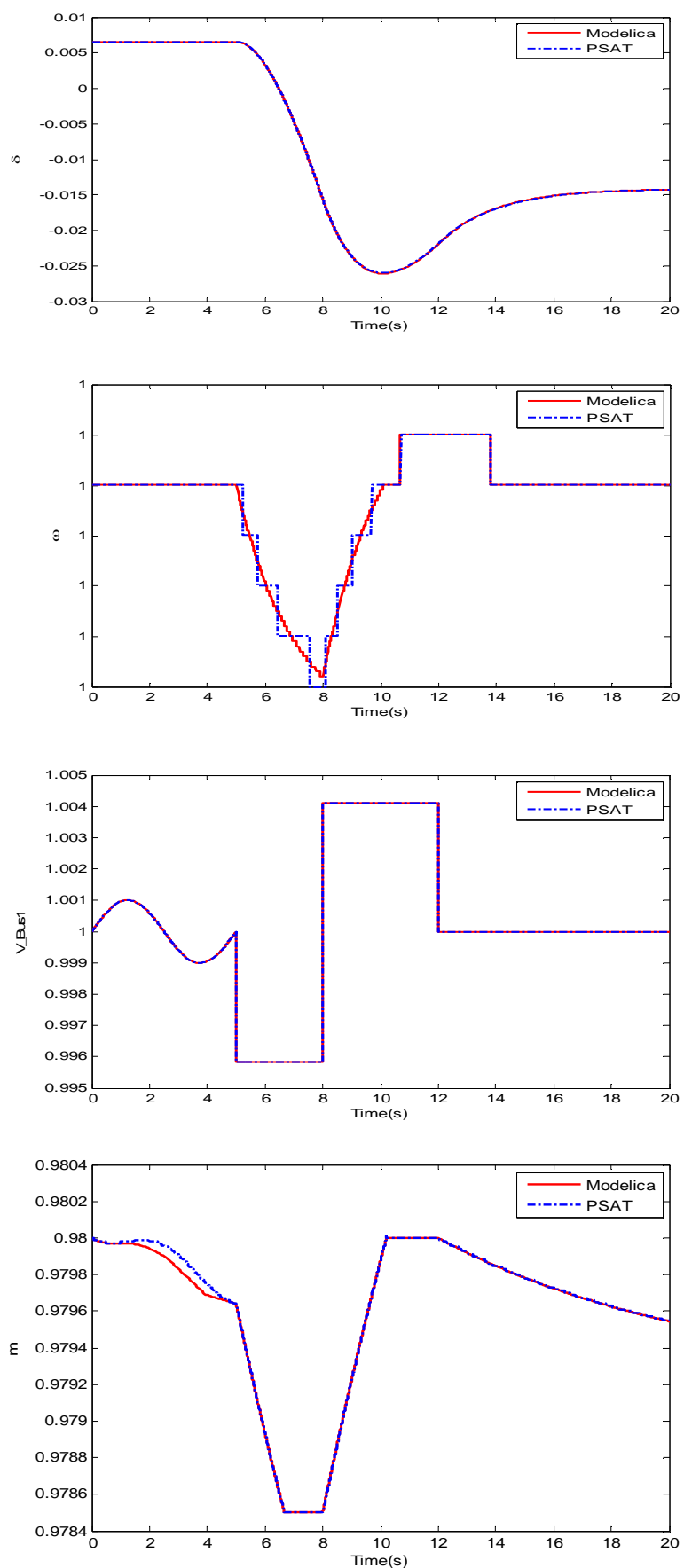

Figure 4: Software-to-Software validation results

\section{REFERENCES}

[1]. C. González. "Power transformer modeling analysis and survey by means of the frequency response”. Master Thesis Dissertation. Carlos III de Madrid University, september 2009.

[2]. Vanfretti, L.; Li, W.; Bogodorova, T.; Panciatici, P., "Unambiguous power system dynamic modeling and simulation using modelica tools," Power and Energy Society General Meeting (PES), 2013 IEEE , vol., no., pp.1,5, 21-25 July 2013.

[3]. P. Fritzson, Introduction to Modeling and Simulation of Technical and Physical Systems with Modelica. Wiley-IEEE Press, 2011. ISBN: 9781-118-01068-6.

[4]. iTesla: Innovative Tools for Electrical System Security within Large Areas. [Online]. Available: http://www.itesla-project.eu/

[5]. F. Milano, Power System Analysis Toolbox Documentation for PSAT. version 2.1.8, 2013.

[6]. D 5.2: Proof of concept for an open simulation and model sharing framework validated on a medium size power system. [Online]: http://fp7-pegase.com/ 